\title{
Kirchliche Kommunikation in der entwickelten Kommunikationsgesellschaft
}

\author{
von Michael Schmolke
}

Gute Sprüche haben ein langes Leben, wenn sie erst einmal eingeschlagen haben. So ist uns allen noch Marshall McLuhan in der Erinnerung und sein "globales Dorf"2. Das war und ist seit 1962 ein Schlagwort so recht nach dem Herzen aller halbwegs gebildeten Menschen. Aber prophetisch war es nicht oder jedenfalls nicht in dem Sinne, den jeder zu verstehen glaubte.

Die Erfindung des Buchdrucks habe zu einer Explosion der menschlichen Rationalität geführt, meinte McLuhan. „Die Produktion von Büchern und Flugschriften beseitigte seiner Ansicht nach endgültig den totalen Dialog des Analphabeten mit seiner geschlossenen, nahen Umwelt der primitiven Dorfgemeinschaft und damit die soziale Harmonie. “3

Das Fernsehen aber erzwinge eine distanzlose, quasi-dörfliche Erlebnisdichte und totale Nachbarschaft und führe in eine längst vergangene Epoche zurück, „indem es auf dem Wege einer neuen Sinneneinheit die abstraktdistanzierte, lediglich visuelle Betrachtung der Umwelt aufhebt: „Die neue elektronische Interdependenz verwandelt die Welt in ein globales Dorf."

Nichts dergleichen ist eingetreten oder einzutreten im Begriff. Im Gegenteil: Je näher uns die Fernseh-Bilder vom zweiten Golf-Krieg oder vom jugoslawischen Bürgerkrieg an die schrecklichen Einzelheiten des Geschehens, manchmal sogar zeitgleich während des Geschehens, heranzwingen, desto deutlicher erleben wir die reale Distanz und den m. E. durchaus berechtigten Wunsch nach Distanz.

Dennoch hat sich die Welt der Kommunikation gewaltig verändert seit McLuhans "Gutenberg Galaxis", und zwar sowohl in jenem Feld, das mein Lehrer Henk Prakke „Zeitgeist und Lebensgefühl“ zu nennen liebte, als auch im Hinblick auf die kommunikative Ausstattung der bewohnten Erde.

Prof. Dr. M. Schmolke ist Professor für Kommunikationswissenschaft und Kommunikationsgeschichte an der Universität Salzburg.

1 Der Beitrag beruht auf einem Vortrag, den der Autor bei einer Akademietagung der Bischöflichen Akademie Aachen anläßlich der Verabschiedung des „Leben und erziehen"-Chefredakteurs Dr. Ferdinand Oertel gehalten hat (August-Pieper-Haus Aachen, 14. November 1992).

2 U. Otto, Rezension von „M. McLuhan, Die Gutenberg-Galaxis“, in: Publizistik 14 (1969), 114-116.

3 Ebd.

4 Ebd.; vgl. Die Gutenberg-Galaxis, 15ff.; $47 \mathrm{ff}$. 
Soweit es um Zeitgeist und Lebensgefühl geht, war McLuhan der Entwicklung voraus. Winfried Schulz, einer seiner Kritiker, schrieb 1969, also vor der „Postmoderne“, über das, was wir heute McLuhans Postmodernismus nennen können: „Die von McLuhan praktizierte nicht-lineare Logik ermöglicht ein voraussetzungsloses Lesen: Man braucht keine Kausalitäten nachzuvollziehen, keine Vorklärungen mitzudenken, man kann sporadisch und punktuell lesen, wenn man will, auch von hinten nach vorn, denn der Text hat so gut wie keine Entwicklung der Argumente, keine gedankliche Hierarchie. ${ }^{5}$ Die nicht-lineare Logik, die wir inzwischen von den New-Age-Autoren, aus der Neo-Esoterik und aus den Guru-Lehren aller Schattierungen bis zum Überdruß kennen, ermöglicht auch - jedenfalls scheinbar - ein „voraussetzungsloses Leben“: ohne Vorklärungen, ohne Kausalitäten glaubt man „sporadisch und punktuell“ leben zu können. Diese Anschauung hat viele Ursachen, aber auch eine wichtige technische Voraussetzung: den kommunikativen Apparat unserer Welt. Er ist inzwischen nahezu omnipräsent.

Der indische Souvenir-Händler, der mir in Fatehpur-Sikri seinen ewigen Kalender verkaufen will, ist aufdringlich und hartnäckig; die Indianerkinder im touristisch aufbereiteten Amazonas-Urwald bieten Blasrohre und Federschmuck an, - mit Charme und Bescheidenheit; die Händlerin auf Seouls Tong-dae-mun-Market thront stolz über Bergen von Frühlingszwiebeln und Trockenpilzen: Gemeinsam ist den dreien der „Getto-Blaster", jenes tragbare Stereo-Monstrum mit doppeltem Kassettendeck, das die Stunden lautstark in Töne verwandelt. Zieht dann die Koreanerin ihr Schnurlos-Telefon unter dem Zwiebelhaufen hervor, um einen Tratsch mit der Kollegin am Ende der kilometerlangen Marktgasse zu beginnen, sieht man als Europäer ganz schön alt aus.

Elektronisch multiplizierte Musik scheint gerade in den Entwicklungsländern allgegenwärtig zu sein; ihre öffentliche Aufdringlichkeit läßt sich aber auch in die Hörknöpfe der Walkmen einsperren, und wo immer wir uns umschauen, sehen wir Kinder, Jugendliche und Erwachsene mit Kopfhörerschmuck. Nur die gelegentlich entweichenden Zischlaute der Rhythmusgruppe erinnern uns daran, daß wir auf Straßen und Plätzen permanent von industriell gefertigter Musik umtönt werden.

Die Omnipräsenz der Medien ist im wörtlichen, wenngleich makabren Sinne, nicht totzukriegen. Während wir uns wundern, daß in Sarajewo, nach inzwischen monatelanger Beschießung, überhaupt noch ein Stein auf dem anderen steht, liefert Radio Sarajewo regelmäßig die neuesten Katastrophenmeldungen. Warum beschießt der Feind niemals die Radiostation? Auch die Fernsehkameras sind oft nahe genug dabei, auf daß wir die Verbohrten und ihre Opfer aus der Nähe sehen können. Die Journalisten bleiben weitgehend unbehelligt, weil die Helden gezeigt und gesendet werden wollen. Ohne Medienpräsenz sind sie politisch nur halb so viel wert.

5 W. Schulz, Rezension von „M. McLuhan, Die magischen Kanäle“, in: Publizistik 14 (1969), 112-114. 
Die Spitzenleistung in dieser neuen Sportart hat bislang der irakische Staatschef Saddam Hussein während des Golfkriegs geliefert. Er hielt sich den amerikanischen CNN-Mann Peter Arnett in seiner Hauptstadt, den Reporter des Satans für die Darstellung der ,Mutter aller Schlachten“ über den TV-Kanal des Erzfeindes. Was hätte ein Goebbels aus den Fernsehen gemacht, hätte er dessen Weiterentwicklung als kriegswichtig durchsetzen können?

Ich male Ihnen diese aus ganz verschiedenen Bereichen stammenden Bilder aus, weil ich damit zunächst einmal meine These illustrieren möchte, die ich Ihnen nicht bzw. nur mit unvertretbar großem Aufwand beweisen kann. Sie lautet: Unsere bewohnte Erde ist durch die Omnipräsenz des elektronisch gestützten Apparats schon heute so verändert, daß die publizistischen und ebenso die pastoralen Ausgangsannahmen des als modern geltenden Zweiten Vatikanischen Konzils nicht mehr stimmen.

\section{Die Kirche versteht die Welt nicht mehr}

Die Kirche sieht sich 75 Jahre nach der Entstehung des Radios und 50 Jahre nach dem Aufkommen des massentauglichen Fernsehens einer globalen Kommunikationssituation gegenüber, die uns, in historischen PrintmedienKategorien gedacht, an den Zustand um die Mitte des 19. Jahrhunderts denken läßt. Zwei in ihren idealtypisch faßbaren Strukturen ähnliche Entwicklungsreihen (in der Zeit) lassen sich parallel stellen:

1. 1450 begann mit der Erfindung des typographischen Druckens die erste Kommunikationsrevolution: Der industriell herstellbare Text für alle wandte sich, propagandistisch gesprochen, schon wenig später auch an alle. Zwischen 1492 und 1555 verändert sich die Welt durch die Entdeckung Amerikas und die Reformation. Beide Innovationen gerieten mit Hilfe des neuen Mediums Buch zu globalen Ereignissen. Hundert Jahre nach Gutenberg reagierte die Kirche mit dem Tridentinum auf die neue Situation. Im 19. Jahrhundert, 400 Jahre nach Gutenberg, machte das nicht mehr neue Medium Presse einen qualitativen wie quantitativen Sprung. Es entstand - cum grano salis: um 1850 - die Massenpresse, der die Kirche aber noch weitere hundert Jahre lang mit den geistigen Mitteln des Tridentinums zu begegnen versuchte.

2. Zwischen 1900 und 1940 traten drei neue Medien auf den Plan: Film, Radio und (aufbauend auf Film und Radio) das Fernsehen.

Zwischen 1945 und 1990 veränderte sich abermals die Welt. Stichworte dazu: Atombombe, Entkolonialisierung, Kalter Krieg, Modernisierung Asiens, Ende des sowjetischen Imperiums und damit Sieg des kapitalistischen über den kommunistischen Materialismus. Schon 25 Jahre nach den ersten regulären Fernsehprogrammen reagierte die Kirche auf die neue Situation: Im Zweiten Vaticanum schuf sie sich neue Instrumente der Zuwendung zur Welt. Hier aber endet die Parallelität. Wie gut oder schlecht die neuen Instrumente zu greifen begonnen haben oder noch greifen werden, dies zu beurteilen, verbieten mir die Grenzen meiner Kompetenz. Eines aber kann ich zuverlässig sagen: Anders 
als bei der ,Zeitreihe I' verlief die Modernisierung der Kommunikationssysteme nicht allmählich bzw. durch deutlich erkennbare Innovationen klar markiert.

Auf der gutenbergischen Zeitachse waren dies nach dem am Anfang stehenden Buch das Aufkommen der Zeitung (1609), der Zeitschrift (1665), der Kolportage-Heftchen (1775), der illustrierten Zeitschrift (1830) und des Taschenbuchs (1860). Die ,Zeitreihe $\mathrm{II}^{*}$ aber kennt nur Innovationsschübe und bislang gar keinen Stillstand. Das gilt für die Kernmedien wie für deren ertragreiche Randzonen, die je für sich techno-ökonomische Imperien darstellen, nämlich Tonaufzeichnung, Bildaufzeichnung sowie Informationsübertragung und -verarbeitung im weitesten Sinne.

Keine Neuerung erlebte ihr quasi natürliches Ende durch echtes Veralten. Beispiele: Kaum war die Schellack-Schallplatte ein Massenprodukt, wurde die Kunststoff-LP serienreif, während beinahe gleichzeitig die magnetische Tonaufzeichnung bereits dem Medium Rundfunk diente. Als um 1930 der Tonfilm mit dem Lichtton-Randstreifen in die Kinos kam, wußten die Techniker schon, daß die Zukunft den magnetisch aufgezeichneten Tönen gehören wird. Während die magnetische Bildaufzeichnung in die Fernsehstudios einzog (Ampex) und schließlich in die Heimelektronik hinüberzuschwappen begann ( Video-Kassette), hatten die Ingenieure von Philips und Sony die Laser-Aufzeichnung von Bild und Ton (CD) bereits fest im Griff. Das jeweils innovative Produkt liegt in den Schubladen und wartet dort, bis der unterhaltungselektronische Markt neuen Schubes bedarf.

Die staatliche Kommunikationspolitik hat sich den Marketingstrategien der Industrie wenn schon nicht gebeugt, so doch geöffnet. Was bei Radio und Fernsehen noch ein zwanzigiähriges Ringen unter den falschen Titeln Rundfunkliberalisierung und Kabelrundfunk gebraucht hat, ging auf anderen Gebieten ganz schnell: Telefone kann man mittlerweile in Deutschland und sogar in Österreich kaufen, statt sie sich verordnen zu lassen. Aber was die Telefonindustrie als den nächsten neuesten Schrei auf dem Lager hat, weiß der Kunde nicht, während er sich bei der Bundespost darauf verlassen konnte, daß es in den nächsten 20 Jahren Änderungen allenfalls bei den Gebühren geben werde.

Der hinhaltende Widerstand gegen die Liberalisierung des SatellitenRundfunks dauerte nur kurz, und der Endverbraucher versteht heute nicht mehr, warum die Verkabelung einst - das ist ganze zehn Jahre her - ein Gegenstand heftigen Streitens war. Bei den Kleincomputern hat gar kein staatlicher oder öffentlich-rechtlicher Regulator aufzutreten versucht, obwohl mir ein solcher gerade hier manchmal sinnvoll erschiene, denke ich an die globalen Netzwerke, die sich z. B. mit Hilfe der Rechenkapazität der Universitäten leicht knüpfen lassen und auf denen heute schon viel Fug und Unfug transportiert wird.

Was das alles mit Kirche und ihrer Kommunikation zu tun habe?

Gestatten Sie mir vorerst eine kurze Zwischenauskunft: Es sind die gleichen Menschen, die sich völlig selbstverständlich in diesem permanent 
expandierenden Kommunikationsumfeld bewegen, alltäglich auf ihre „messages" warten und zugleich die Adressaten der Botschaft sind.

Ehe ich mich dem Einwand mit der gebotenen Sorgfalt widme, möchte ich jedoch meine These, über das illustrative Verfahren hinausgehend, mit zwei Zeugenaussagen stützen, die jede für sich hohe Glaubwürdigkeit beanspruchen dürfen; beide stammen aus jüngster Zeit (22. bzw. 26. Oktober 1992), geben also das aktuelle Urteil ihrer Autoren wieder. Es handelt sich dabei um den Zürcher Kommunikationswissenschaftler Ulrich Saxer und um Gerd Bacher, den Generalintendanten des ORF.

\section{Die kommunikative Umwelt: allgegenwärtig und überkomplex}

Ulrich Saxer systematisiert die von mir z. B. in Bildern beschriebene Situation folgendermaßen: „Im 20. Jahrhundert entwickeln sich im Westen aus industrialisierenden industrialisierte und seit den 60er Jahren postindustrielle bzw. Informationsgesellschaften. Weltweit bestehen gegen Ende des Jahrhunderts diese drei Gesellschaftstypen nebeneinander. [...] Charakteristisch für Industriegesellschaften ist die durch Großorganisationen bewerkstelligte Massenkommunikation zur Etablierung und Stabilisierung von Massenmärkten, während die Komplexität postindustrieller Gesellschaften einen solchen Grad erreicht, daß immer spezialisiertere Medienkommunikation die darin exponentiell anwachsenden unterschiedlichen Kommunikationsbedürfnisse absättigen muß. Dies erfordert den Aufbau eines sogenannten Quartärsektors Information, aber auch die politische Dezentralisierung der Medienkontrolle, flexible Organisationsstrukturen, um ein rasches Eingehen des Quartärsektors auf die Umweltbedürfnisse zu gewährleisten, setzt überhaupt der Durchregelung Grenzen und muß sich demzufolge auf eine entwickelte, eigenständige Journalismuskultur stützen können.“

Zugleich erkennt Saxer „die Schwierigkeit, daß im Prozess evolutionärer Differenzierung dieser Gesellschaft, der immer neue Berufe, wissenschaftliche Disziplinen, Dienstleistungen etc. hervorbringt, der Quartärsektor, der den Tertiärsektor kommunikativ erschließen sollte, selber überkomplex wird, so daß immer häufiger, von Tschernobyl bis zum Golfkrieg, Informationschaos droht."

Der Medienpraktiker Gerd Bacher vollzieht für sich die gleiche Analyse, bedient sich jedoch der journalistischen Sprache: „Als vor 25 Jahren, der neue, der moderne ORF begann, herrschte noch technische Steinzeit. Heute beschäftigen sich seine Fachleute mit avancierten Techniken, die zu exotisch sind, um sich ihre Laborbezeichnungen zu merken. Dazwischen liegen 25 Jahre technische Revolutionen, wie sie die Zivilisationsgeschichte in dieser Dichte und in diesem Tempo nie erlebt hat. Arbeits- und Lebenswelt wurden im Verlauf dieser 25 Jahre teilweise auf den Kopf gestellt. Unsere kulturellen Reflexe,

6 Saxer, Medienwandel - Journalismuswandel. Referat auf dem Symposion „Ein Jahrhundert(-)Journalismus 1900-2000", Wien 22. Oktober 1992. Msk. 
Prägungen und Ideale, die vielfach im 19. Jahrhundert wurzeln, stießen in diesen 25 Jahren immer häufiger auf die Technologie des 21. Jahrhunderts. Kollisionskurs ist zu orten. [...] [Früher] konnten wir noch guten Gewissens von der Vorstellung ausgehen, daß der Zentral-Fundus hehren abenländischen Kulturguts von Radio und Fernsehen bloß verbreitet werden müsse, und es würde Licht werden im Bewußtsein der Bürger, die es nach solcher Beglückung dürste. Inzwischen ist die Sache sehr viel komplexer, auch verwirrender geworden. Immer weniger ist die Entdeckung zu verdrängen, daß die elektronischen Medien selbst zu den bestimmenden, epocheprägenden Kulturphänomenen geworden sind. Die Alltagskultur ist von nichts so stark geprägt, durchdrungen und wohl auch beschädigt wie vom Dauerbombardement elektronischer Bilder und Töne. Und Kunst und Philosophie unserer Tage handeln von nichts anderem so sehr wie vom Versuch, mit den Folgen dieser nie dagewesenen Reizüberflutung moralisch, ästhetisch, emotional und intellektuell fertigzuwerden. Medien nicht nur als Kennzeichen, sondern als Schicksal der nach ihnen benannten Gesellschaft."

Dieser Dramatisierung wird sich nicht jeder anschließen mögen, der Zustandsbeschreibung und -analyse wird man sich jedoch kaum entziehen können.

In die also beschriebene Welt hinein erläßt nun im Juni 1992 die Kongregation für die Glaubenslebre eine „Instruktion über einige Aspekte des Gebrauchs der sozialen Kommunikationsmittel bei der Förderung der Glaubenslehre". Sie erinnert an Imprimatur und Nibil obstat und weist auf die im CIC vorgesehenen „Verwaltungs- oder Strafmaßnahmen“ hin. ${ }^{8}$ Ich habe nicht vor, jetzt in eine kleinliche Instruktionsschelte einzutreten. Die neue Instruktion diene lediglich als Exempel. Während sich die Weltbevölkerung offensichtlich dem neuen, global durchschlagenden Kommunikationsstil zu unterwerfen anschickt und in zahlreichen Gesellschaften bereits einen veränderten Kommunikationshabitus angenommen hat, sorgt sich die Kongregation für die Glaubenslehre darum, daß „durch die sozialen Kommunikationsmittel im allgemeinen und durch die Bücher im besonderen heute immer mehr irrige Gedanken verbreitet" werden.

Das Kommunikationsproblem der Kirche ist aber doch nicht, daß die zur Überwachung verpflichteten Oberhirten mit deren Durchführung Schwierigkeiten haben, das Problem ist nicht einmal, daß ,immer mehr irrige Gedanken verbreitet" werden, sondern daß eigentlich alles überall und ständig verbreitet wird und daß in diesem Totale notwendig auch die irrigen Gedanken reichlich vertreten sind. „Droht uns“, fragt Gerd Bacher, „statt des nuklearen

7 Bacher, 25 Jahre ORF. Rede bei der Jubiläumsfeier am 26. Oktober 1992 im ORFZentrum Wien. Msk.

8 Kongregation für die Glaubenslehre: Instruktion über einige Aspekte des Gebrauchs der sozialen Kommunikationsmittel bei der Förderung der Glaubenslehre (Rom, 30. März 1992). Abdruck in: Pressemitteilungen der Deutschen Bischofskonferenz, 12. Juni 1992. 
Winters der nie endende mediale Winter? Es wird unausgesetzt und immer noch mehr Bilder und Töne schneien." Er erweckt Reue: „Wir Bilder- und Töne-Produzenten sind nicht mehr nur Zeugen, wir sind vor allem auch Täter. Wir haben im letzten Vierteljahrhundert die Atmosphäre mit verführerischen, aber auch mit gewalttätigen und obszönen Codes geradezu gesättigt."

\section{Erinnerung an die „zweite Kanzel“}

Wie schafft es die Kirche, mit ihrer Botschaft inmitten der „Täter“ zu bestehen und ibre Codes unter die Leute zu bringen?

Die klassische Methode, nämlich die Verkündigung in Kirche und Schule, gibt schon lange nicht mehr genug her. Schon sehr früh wurde daher die Presse als „zweite Kanzel“ in Pflicht genommen. Der Mainzer „Katholik“ kann als Erfinder des neuen Weges gelten. 1844 schrieb er: „Was sie nicht mehr am Fuße der heiligen Tribüne hören, das müssen sie in Werken von jedem Formate lesen können, das müssen sie in periodischen Schriften finden, das müssen ihnen die Tageblätter zu jeder Tageszeit in ihre Geschäftszimmer, in die Werkstätte des Arbeiters, in die Versammlungen zum Vergnügen und sogar auf ihren Nachttisch bringen".?

Aber der neue Weg erwies sich je länger, je mehr als Holzweg. Heute, wo sich nur mehr 22 Prozent der deutschen Katholiken regelmäßig „am Fuße der heiligen Tribüne" versammeln, ist die Frage nach anderen Wegen bis zum Überdruß ausdiskutiert. Alle Verbesserungen der kirchlichen Medienpublizistik haben nämlich wenig gefruchtet.

Das Problem, so setze ich meine These fort, liegt nicht beim Medium, sondern beim Publikum.

Man mißverstehe diese Achsendrehung nicht als einen dialektischen Trick. Ich will jetzt auch nicht aus der Einsicht heraus, daß alle Modernisierungsanstrengungen $z$. B. bei der Kirchenpresse die Auflagen-Talfahrt nicht haben stoppen können, den Schwarzen Peter den Lesern zuschieben. Ich gehe vielmehr davon aus, daß das Publikum der 90er Jahre ganz andere Rezeptionsgewohnheiten hat als jenes der 50er Jahre. Es hat sich umgewöhnt, es hat gelernt, es hat sich umschulen lassen.

Man weise ihm also nicht generell die Schuld zu, etwa mit Sätzen wie: das Publikum wolle nicht mehr hören oder es könne nicht mehr hören, was ihm die Kirche zu sagen habe, es sei verbildet, verführt, verblendet.

Treffend scheint mir nur eine deutschsprachige Wendung den derzeitigen Zustand der Aufnahmebereitschaft und -kapazität zu erfassen, eine Wen-

9 Vgl. M. Schmolke, Die schlechte Presse, Münster/W. 1971, 312. 
dung, die aus dem Jugend-Jargon kommt: ein großer Teil jedenfalls des europäischen Publikums „hat keine Rille" mehr für die Botschaft der Kirche.

Die aus der Technologie der Analog-Schallplatte entlehnte Metapher von der „Rille“ bildet das Gemeinte ziemlich genau ab. Es geht nämlich um Kapazität, Inhalt und Form.

Was die Form angeht, gilt am ehesten die Beobachtung, daß wir es mit "umgeschulten" Empfängern zu tun haben: Entertainment bzw. Infotainment ist das Schlagwort für die Gestaltungsweise, die Akzeptanz verspricht. Die konventionelle Schule steht vor dem gleichen Vermittlungsproblem; wir alle kennen die peinlichen Anbiederungsversuche mit künstlichen Aktualisierungen und Primitivisierungen - als ob man jede Wahrheit auch als Comic-Serie vermitteln könnte.

Was die Kapazität angeht, so wußte die Kirche schon früher, daß ihr Gesamtangebot zu umfangreich ist; daraus ist der Kleine Katechismus entstanden. Aber auf Spot-Längen, die heute jeder zu schlucken gewohnt ist, läßt er sich dann doch nicht reduzieren.

Von den Inhalten zu reden, bedeutet, ein weites Feld zu eröffnen; denn die Inhalte sind noch am ehesten geeignet, der Botschaft Zugang zu verschaffen. Da sind die großen Fragen nach dem Leben und dem Tod und dem Leben nach dem Tod, nach Glück und Schuld und dem Umgang mit ihnen, nach Krankheit, Verzweiflung und Hoffnung. Wir müssen den Medien einräumen, daß sie zu diesen Fragen auch die Kirche hören und bewußt zu Wort kommen lassen.

Aber eben nur "auch". Oder genauer beobachtet: Die Dramaturgie der Medien, keineswegs nur des Fernsehens, verleiht der jeweiligen kirchlichen Aussage gerade durch dieses „auch“, durch das Zu-Wort-Kommen in Konkurrenz, im Widerspruch, im Konflikt, jenes Gewicht, das zu Respekt oder, im ebenfalls möglichen Falle des Versagens, zur Lächerlichkeit führt.

Dort aber, wo die Kirche vom reservierten Platz aus spricht, entzieht sie sich der medieneigenen Dramaturgie, die Spannung bleibt aus und damit auch die Zuwendung von Aufmerksamkeit.

Wer dieser Beobachtung zustimmt und sich etwa zu dem Satz versteht: „Ein streitbarer Bischof in einer Talk-Show ist mehr wert als hundert Worte zum ,Sonntag'“, sitzt selber schoñ auf dem neuen Zug, - man vergesse doch bitte nicht, daß die Kirche mit dem Ausschließlichkeitsanspruch der Verkündigung der einen richtigen Lehre angetreten ist und sich nicht damit zufrieden geben darf, daß sie „auch" zu Wort kommt, und zwar lediglich um der Spannung und der Aufmerksamkeit willen.

Das um der Anpassung willen betriebene Mitgehen im Kommunikationsstil der Zeit darf also ihre Sache nicht sein - oder allenfalls nebenbei, nicht im Wesentlichen. 


\section{Konflikt-Themen als Einflugschneise?}

Immerhin kann man aus der medialen Konflikt-Dramaturgie, wie sie zuerst wohl die „Spiegel“-Gespräche entwickelt haben, wie sie im Fernsehen der frühe „Club 2" des ORF auf ein spannungsreiches Niveau gebracht, wie sie schließlich der „Heiße Stuhl“ bis zur Selbst-Persiflage überdreht hat, lernen, daß der konfliktbezogene Inhalt als solcher durchaus den Durchbruch zum Publikum der 90er Jahre schaffen kann. Das gilt auch, wenn er ohne Mätzchen dargeboten wird. Kirchliche Kommunikation aber scheut, wenn es sich nicht gerade um Apologetik im wörtlichen Sinne handelt, die konfliktbezogenen Themen.

Es gibt Ausnahmen. In einer katholischen Familienzeitschrift vom November 1992 lese ich folgende Geschichte: „Als ich von meiner Schwangerschaft erfuhr, war ich zunächst einmal schockiert', erinnert sich Mona Gisbert. Denn was nun auf sie zukam, mußte sie allein mit sich aushandeln. ,Marc, den Vater meines Kindes, wollte ich nicht heiraten. Die Beziehung wäre sowieso über kurz oder lang in die Brüche gegangen', erzählt sie. Mona zog den Schlußstrich - die ,im Nachhinein einzig richtige Entscheidung “ " "10

Ein Redakteur, der eine so beginnende Geschichte 1950 in der „Kirchenzeitung für das Erzbistum Köln“ hätte durchgehen lassen, wäre umgehend gefeuert worden. Angenommen, er hätte sich brav wieder hochgedient und wäre 1961 Chefredakteur der in Essen erscheinenden "Christlichen Familie" geworden und hätte es dann noch einmal versucht mit diesem flott geschriebenen Manuskript, so hätte ihm der in diesem Falle nicht einmal kirchen-offizielle Verleger ebenfalls nahegelegt, sich einen Schreibtisch in der damals noch existierenden „Constanze"-Redaktion zu suchen. Hätte der Verleger ihn unvorstellbarerweise gehalten, so wäre dem katholischen Deutschland in der nächsten Ausgabe der „Neuen Bildpost“ die Schlagzeile präsentiert worden: „Christliche Familie propagiert freie Liebe“. Spätestens jetzt hätte Bischof Hengsbach den jungen Mann zu einem Gespräch eingeladen, und der Chefredakteur des nördlich von Essen ansässigen größten Bistumsblattes deutscher Zunge hätte die jungen Herren seiner Redaktion für 11.55 Uhr zu einer kurzen Vergatterung gebeten, - solche Geschichten gebe es zwar und er könne da dies und jenes aus Warendorf dazu beitragen, das gehöre in camera caritatis behandelt, aber nie und nimmer in den Spalten von ... - und damit wäre er ohne weiteren Kommentar zum Vorbeten des „Engel des Herrn“ übergegangen, denn es war inzwischen $12 \mathrm{Uhr}$.

\section{Alles besser, aber keine Remedur}

Jetzt wissen Sie alle, daß ich im letzten Teil meines Vortrags auf den biographischen Anlaß dieser Akademietagung einzugehen gedenke. Der erwähnte Bischof und auch der vorbetende Chefredakteur sind nicht mehr unter uns, aber jenen Redakteur bzw. später Chefredakteur gibt es glücklicherweise

10 M. N.; Neun Monate ganz allein?, in: Leben und Erziehen Nr. 11/1992, $20 \mathrm{f}$. 
noch und auch jene Zeitschrift, in der die Jung-Mutter Mona von ihren "Neun Monaten ganz allein“ erzählt. Fingiert ist die Annahme, daß das zugrundeliegende Manuskript 1951 oder 1961 auf einen katholischen Redaktionsschreibtisch hätte gelangen können. Undenkbar. Die Inhalte der je Medientyp denkbaren Texte haben sich zwischen 1950 und 1990 nämlich genau so verändert wie die Kommunikationsgesellschaft. Verändert haben sich natürlich auch die Menschen, die von Berufs wegen mit Manuskripten umzugehen haben, und wenn schon nicht die Menschen, so doch ihre Maßstäbe und Urteile.

Wer wie Ferdinand Oertel seit 40 Jahren schreibt und redigiert, hat die Veränderung der Kommunikationswelt, die ich zu skizzieren versucht habe, in vollem Umfang miterlebt, und zwar ohne Netz und doppelten Boden und ohne Narkose. Wer die Stationen seines Lebenslaufes bedenkt, sieht, daß er immer Täter war, aber für viele Jahre zugleich Beobachter. Beobachter, Kritiker und Analysator als Agentur-Journalist, AKP-Funktionär und UCIP-Funktionär, als Beraur der Bischofskonferenz, des Zentralkomitees der deutschen Katholiken und der Würzburger Synode. Ein Berater, der auf der Grundlage des Nachdenkens über den eigenen Beruf Rat gegeben hat, der aber reflektierend in der Lage war, sein Metier und seine Branche in Frage zu stellen. Unter den Redakteuren der Kirchenpresse war er kein enfant terrible, gehörte wohl gerade noch zum mainstream, machte aber gelegentlich Vorschläge, die von der Mehrheit nicht als verwirklichenswert eingestuft wurden.

Auch ich bin nicht sicher, ob seine durch selbstkritische Reflexion getesteten Vorschläge wirklich Remedur geschaffen hätten. Denn in seinem Berufsweg und in den Aufgaben, die er sich freiwillig aufgeladen hatte, spiegelt sich das Dilemma, das ich im ersten Teil herauszuarbeiten versucht habe: die ihm zunächst unbewußte, vielleicht erst in den Jahren bei „Leben und Erziehen“ bewußt werdende Erfahrung, daß die Adressaten und ihre Welt sich verändern, während die Botschaft die gleiche bleiben muß. Und daß deshalb bei den Hütern der Botschaft Unsicherheit entsteht und bei den Vermittlern Resignation: nach jahrzehntelangen Anstrengungen, es mit neuen Kleidern zu versuchen.

Der Schlußsteil meines Vortrags gerät also nicht unversehens zur Laudatio, sondern zur Geschichte einer Ohnmacht. Ferdinand Oertel hat in 40 Berufsjahren eine große Zahl positiver Veränderungen und Neuerungen in Kirche und kirchlicher Publizistik miterlebt, zum Teil selber angestoßen. Auf der Kommunikator-Seite ist beinahe alles, der Papierform nach, besser geworden:

- Die Bistumsblätter sehen wie Magazine oder wie zeitgemäße Wochenzeitungen aus,

- die Redaktionen sind viel stärker und überwiegend mit Journalisten statt mit Kaplänen besetzt,

- die Katholische Nachrichtenagentur ist eine modern ausgestattete Spezialagentur geworden,

- die Deutsche Bischofskonferenz hat, wie alle Diözesen auch, eine funktionstüchtige Pressestelle, 
- Marketing und Betriebswirtschaft werden, seitdem es die Mediendienstleistungsgesellschaft (MDG) gibt, nicht mehr klein geschrieben,

- die Kirche selbst hat sich im Zweiten Vaticanum neue Interpretationen und Kategorien und sogar erste Ansätze einer dem 20. Jahrhundert angemessenen Sprache zugelegt,

- die Kirche in Deutschland hat mit der Würzburger Gemeinsamen Synode (1971-1975) eine geradezu unverhältnismäßige Anstrengung unternommen, mit "unserer Zeit" ins Gespräch zu kommen,

- einige Diözesen haben, nachdem der gemeinsame Versuch mit der Wochenzeitung „Publik“ gescheitert war, durch das permanente Engagement beim „Rheinischen Merkur" wenigstens eine große, überregional gehörte Stimme zu erhalten versucht,

- die Unternehmen der Kirchenpresse haben in der AKP einen hohen Organisationsgrad erreicht und immer wieder Solidarität bewiesen, auch wenn es um heikle Selbstanalyse ging,

- nicht wenige Unternehmen haben radikale Modernisierungen gewagt, mit Beilagen experimentiert, sich zu Kooperationen entschlossen.

Aber auf der Publikums-Seite ist es trotzdem schlechter geworden; am Anfang der 90er Jahre wollen weniger Menschen zuhören und lesen als am Anfang der 80er, und damals waren es weniger als am Anfang der 70er.

1992 erscheint ein Buch von Heiner Boberski, Mitarbeiter auch der Oertel-Festschrift, mit dem Titel „Die Divisionäre des Papstes“11, in dem der „Furche"-Redakteur die „Bischofsernennungen unter Johannes Paul II.“ behandelt und die Befürchtung ausdrückt, daß allein schon die Personalpolitik des Papstes die Botschaft konterkariert, wie sie vom postmodernen und - in nicht wenigen Entwicklungsländern - vom prämodernen Menschen verstanden werden könnte.

1992 bescheinigt sich das Freiburger Diözesanforum, es „habe die Kirche nicht mehr als Apparat erscheinen lassen, sondern als eine Kirche, die nach dem Vorbild Jesu den Menschen und ihren Lebensprozessen wieder unmittelbar nahe ist. ${ }^{12}$ Da sprechen Insider einander Mut zu - hoffentlich hilft es, vielleicht auch nach außen.

\section{Postmodernismus, Postmaterialismus, Wertewandel}

Außen, aber nicht unbedingt draußen, steht eine Gesellschaft, die mit dem Etikett des Postmodernismus nicht zulänglich beschrieben ist. Denn erstens ist diese Kategorie ohnehin nur auf den Lebensstil hochindustrialisierter, reicher Länder anwendbar und zweitens wird ein Denk-Kaleidoskop, daß durch Eklektizismus, durch Formeln wie "unübersichtliche Gesellschaft" und durch eine Art Hypertoleranz gekennzeichnet ist, die widersprüchliche Wertsy-

11 H. Boberski, Die Divisionäre des Papstes, (Otto Müller-Verlag) Salzburg 1992.

12 KNA-II), 5. November 1992, 14847. 
steme nebeneinander gelten läßt und keine Perspektive für die weitere Entwicklung der Menschheit eröffnet.

Wesentlich ernster zu nehmen sind jene soziologischen Langzeitstudien, die dem Wertewandel gelten. Religiöse Werte spielen in ihnen eine wichtige Rolle.

Deren tiefe Verankerung und Langlebigkeit könnte Anlaß zur Freude sein. Aber schon der Befund, daß postmaterialistische Wertvorstellungen auf dem Vormarsch sind, ist geeignet, Verwirrung zu stiften, denn post-materialistisch heißt keineswegs nicht-materialistisch oder gar idealistisch. Es sind die im Rückzug befindlichen klassischen Materialisten (und nicht die Postmaterialisten), die „viel häufiger ... an den traditionellen jüdisch-christlichen Normen" festhalten. ${ }^{13}$

Verkehrte Welt? Nein, sie - die Materialisten - hatten in Zeiten der Unsicherheit das größte Bedürfnis „nach der Orientierungshilfe und dem Rückhalt, die vertraute kulturelle Normen und absolute religiöse Glaubensvorstellungen bieten können". ${ }^{14}$

Hier liegt zweifellos ein Ansatzpunkt soziologisch orientierter Pastoral, die sich in Zukunft um die Wiederbelebung der Kommunikation zu kümmern haben wird.

Der zur Zeit in der zweiten und dritten Welt viel rapider ablaufende Wertewandel entzieht sich vorerst den Kategorien der europäisch-amerikanischen Wertwandelforschung.

Das einzige, was beinahe überall gleich rasch voranschreitet und sich relativ leicht beobachten und messen läßt, was also auch ein Tertium comparationis abgibt, ist der Prozeß, der zur entwickelten Kommunikationsgesellschaft führt. In ihm inhaltlich vertreten zu bleiben, müßte Hauptziel kirchlicher Kommunikation sein. Fragen Sie mich nicht, wie das geschehen und wer da verantwortlich sein soll. Mit Instruktionen allein wird es nicht zu machen sein, und auch die Divisionäre des Papstes scheinen kein Patentrezept zu haben, weder in Sankt Pölten noch in Santo Domingo.

13 Inglehart, Kultureller Umbruch. Wertwandel in der westlichen Welt, Frankfurt/M. 1989, 235.

14 Ebd. 


\section{SUMMARY: Ecclesiastical communication in the developed communi- cation society}

At the end of the second millenium, modern communication networks are spread throughout the populated world. It is disputable whatever the omnipresence of communication technology and the permanent availability of the offers of the mass media are more useful or more damaging to the development of mankind. Yet, one cannot deny that people naturally turn towards these offers and make use of them.

Whenever the church wants to communicate with mankind, it encounters this state of complete communicative equipment natured within the last 40 years. It does so in any case in industrial countries and in countries embarking on this state. Both the equipment and the technologically determined style and rite of communication are already there, where the church wants to catch on with its message. But just as the church reacted too late first to Gutenberg and then to the mass press coming into existence in the 19th century, its reaction to the present electronic revolution in communication is not comprehensive nor competent enough. The modernisations of ecclesiastical speech and mass media are indeed remarkable. But they have little effect on a public which is no longer able to hear the one message well, because it is used to hear many different „messages“ every day. The church has to align all its efforts with upholding the representation of its content within the process which leads to a developed communication society. This can only succeed if it notes that the people living in this communication society are the way they are and if it is prepared to translate this knowledge into pastoral practice.

\section{RÉSUMÉ: La Communication religieuse dans une societé de communi- cation développée}

A la fin du deuxième millénaire, des réseaux modernes de communication s'étendent sur toutes les parties habitées de la terre. On peut discuter sur le fait de savoir si l'omniprésence des technologies de communication et la possibilité d'avoir recours en permanence aux moyens audio-visuels profitent ou nuisent à l'évolution de l'humanité. Mais on ne peut pas contester le fait que les hommes se tournent sans préjugé vers ces offres et les utilisent.

A chaque fois que l'Église veut communiquer avec l'humanité, elle se trouve, en tout cas dans les pays industrialisés et dans ceux en passe de le devenir, face à cet état d'équipement complet en matiére de communication, un état qui a été atteint grâce au développement des 40 dernières années. Aussi bien l'équipement que certains styles et habitus techniques de communication sont déjà présents, là où l'église veut faire passer son message. Mais, come l'église avait autrefois réagi trop tard à Gutenberg et après, au 19 è siècle, à l'apparition de la presse de masse, de même, aujourd'hui, elle ne réagit pas de façon suffisament vigourouse et compétente face à la révolution électronique dans le domaine de la communication. Il est vrai que la modernisation du langage religieux et celle des moyens audiovisuels religieux sont remarquables. Mais elles n'ont pas assez de résonance face à un public qui ne peut plus très bien entendre ce message parce qu'il est habitué à entendre tous les jours beaucoup de messages différents.

Dans le procès qui conduit à la société de communication développée, l'Église doit s'efforcer de rester représentée par ses contenus. Ceci ne peut réussir que si elle prend acte que les hommes de la société de communication sont comme ils sont et que si elle est prête à mettre cette constatation en pratique au niveau pastoral. 
RESUMEN: La comunicación de la Iglesia en una sociedad de la comunicación desarrollada

A fines del segundo milenio, las redes de comunicación moderna cubren totalmente la tierra habitada. Se puede discutir si la presencia de las técnicas de comunicación y la permanente disponibilidad de las ofertas de los medios de comunicación de masas promueven o dañan el desarrollo de la humanidad. Pero no se puede negar que los hombres ponen atención a estas ofertas en forma ingenua y las usan.

Siempre que la Iglesia quiere comunicarse con la humanidad, encuentra, por lo menos en los países industrializados y en los que se encuentran en su umbral, un estado de equipamiento completo en lo que toca a la comunicación que fue madurando en los últimos 40 años. Tanto el equipamiento como el hábito y estilo de la comunicación tecnicamente determinados, están presentes allí donde quiere llegar la iglesia con su mensaje. Pero, así como la iglesia reaccionó tardíamente, primero respecto a Gutenberg y después en el siglio XIX frente a la naciente prensa de masas, hoy reacciona a la revolución de la comunicación electrónica de forma no global, ni suficientemente competente. La modernización del leguaje eclesial y de los medios de masas eclesiales son dignos de mención. Pero tienen poco efecto frente a un público que no puede escuchar bien la una mensaje porque está acostumbrado a oír todos los dias diferentes mensajes. La Iglesia debe orientar todos sus esfuerzos a permanecer presente con sus contenidos en el proceso que lleva a la sociedad de la comunicación desarrollada. Y en ésto sólo tendrá éxito si toma en consideración que los hombres de la sociedad de la comunicación desarrollada son tal como son, y si está dispuesta a llevar a efecto este conocimiento a la práctica pastoral. 